Technische

Universität

Berlin

\author{
Michael Stöhr, Kilian Oberleithner, Moritz Sieber, Zhiyao Yin, \\ Wolfgang Meier
}

\title{
Experimental study of transient mechanisms of bistable flame shape transitions in a swirl combustor
}

Open Access via institutional repository of Technische Universität Berlin

\section{Document type}

Journal article | Accepted version

(i. e. final author-created version that incorporates referee comments and is the version accepted for publication; also known as: Author's Accepted Manuscript (AAM), Final Draft, Postprint)

This version is available at

https://doi.org/10.14279/depositonce-12718

Citation details

Stöhr, M., Oberleithner, K., Sieber, M., Yin, Z., \& Meier, W. (2017). Experimental Study of Transient Mechanisms of Bistable Flame Shape Transitions in a Swirl Combustor. In Journal of Engineering for Gas Turbines and Power (Vol. 140, Issue 1). ASME International. https://doi.org/10.1115/1.4037724.

Terms of use

This work is protected by copyright and/or related rights. You are free to use this work in any way permitted by the copyright and related rights legislation that applies to your usage. For other uses, you must obtain permission from the rights-holder(s). 


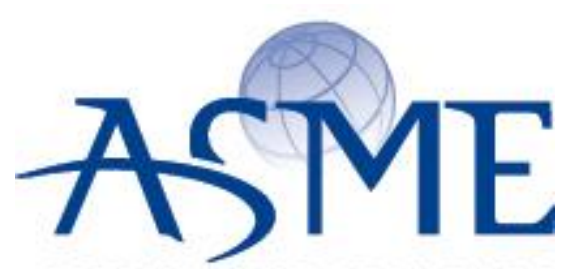

\section{American Society of Mechanical Engineers}

SETTING THE STANDARD

ASME Accepted Manuscript Repository

Institutional Repository Cover Sheet

ASME Paper Title: Experimental Study of Transient Mechanisms of Bistable Flame Shape Transitions in a

Swirl Combustor

Authors: $\quad$ Michael Stöhr, Kilian Oberleithner, Moritz Sieber, Zhiyao Yin, Wolfgang Meier

ASME Journal Title: Journal of Engineering for Gas Turbines and Power

Volume/Issue 140(1): 011503 (8 pages)

Date of Publication (VOR* Online) September 19, 2017

ASME Digital Collection URL: https:// asmedigitalcollection.asme.org/gasturbinespower/

article-abstract/140/1/011503/374503/

DOI: $\quad$ https://doi.org/10.1115/1.4037724

*VOR (version of record) 


\author{
Proceedings of the ASME Turbo Expo 2017: Turbomachinery Technical Conference \\ and Exposition \\ GT2017 \\ June 26-30, 2017, Charlotte, USA
}

GT2017-65003

\title{
EXPERIMENTAL STUDY OF TRANSIENT MECHANISMS OF BI-STABLE FLAME SHAPE TRANSITIONS IN A SWIRL COMBUSTOR
}

\author{
Michael Stöhr* \\ German Aerospace Center (DLR) \\ Institute of Combustion Technology \\ Pfaffenwaldring 38-40 \\ 70569 Stuttgart, Germany
}

\author{
Kilian Oberleithner, Moritz Sieber \\ Chair of Fluid Dynamics \\ Hermann-Föttinger-Institut \\ Technische Universität Berlin \\ Müller-Breslau-Str. 8 \\ 10623 Berlin, Germany
}

\author{
Zhiyao Yin, Wolfgang Meier \\ German Aerospace Center (DLR) \\ Institute of Combustion Technology \\ Pfaffenwaldring 38-40 \\ 70569 Stuttgart, Germany
}

\begin{abstract}
Sudden changes of flame shape are an undesired, yet poorly understood feature of swirl combustors used in gas turbines. The present work studies flame shape transition mechanisms of a bistable turbulent swirl flame in a gas turbine model combustor, which alternates intermittently between an attached $V$-form and a lifted $M$-form. Time-resolved velocity fields and $2 D$ flame structures were measured simultaneously using high-speed stereo-PIV and $O H-P L I F$ at $10 \mathrm{kHz}$. The data analysis is performed using two novel methods that are well adapted to the study of transient flame shape transitions: Firstly, the linear stability analysis (LSA) of a time-varying mean flow and secondly the recently proposed spectral proper orthogonal decomposition (SPOD). The results show that the transitions are governed by two types of instability, namely a hydrodynamic instability in the form of a precessing vortex core (PVC) and a thermoacoustic (TA) instability. The LSA shows that the V-M transition implies the transient formation of a PVC as the result of a self-amplification process. The $V-M$ transition, on the other hand, is induced by the appearance of a TA instability that suppresses the PVC and thereby modifies the flow field such that the flame re-attaches at the nozzle. In summary these results provide novel insights into the complex interactions of TA and hydrodynamic instabilities that govern the shape of turbulent swirl-stabilized flames.
\end{abstract}

\footnotetext{
*Address all correspondence to this author: michael.stoehr@dlr.de
}

\section{INTRODUCTION}

Modern gas turbines are required to run at low NOx emissions and over a wide range of operating conditions. Rapid mixing of the reactants downstream of the fuel injection and robust flame stabilization mechanisms are strong requirements for the design of new combustors. Swirl-stabilized combustion is currently the most common approach. Strong swirl is imparted on the combustor flow to enhance turbulent mixing and to generate an internal recirculation zone (IRZ) which acts as an obstaclefree flame holder.

In an ideal case the turbulent swirl flame remains well anchored throughout a wide range of operating conditions and its shape changes only gradually within this range. In practice, however, swirl flames often exhibit abrupt changes of flame shape at certain operating conditions as reported, e.g., in Refs. [1-6]. This is highly undesired since it may lead to a sudden changes of emissions or thermoacoustic pulsations, or to thermal stresses of combustor walls due to abrupt changes of local temperature. When a flame is operated near the point of shape transition, it may further exhibit a so-called bi-stable behavior where the flame alternates intermittently between two shapes without external influence.

While a few studies of bi-stable flames have been reported [2,5-7], the detailed mechanisms remain largely unclear since they take place on short time-scales and involve complex interactions of turbulent flow field and chemistry. Additional knowledge about the underlying mechanisms may help to design im- 
proved combustors with an extended range of stable operation. The present work therefore aims at a detailed experimental investigation of flame shape transitions using time-resolved multiparameter diagnostics and advanced methods of data analysis.

The work continues the previous study by Oberleithner et al. [6] focusing on the formation and suppression of a helical coherent flow structure, commonly known as the precessing vortex core (PVC), in a swirl combustor. The authors performed a linear stability analysis (LSA) of the time-averaged reacting flow fields. They investigated two different reacting operating conditions, one with an attached conical V-shaped flame with a strong density stratification at the combustor inlet and one with a detached M-shaped flame with mild density stratification at the inlet. Their analysis showed that the strong density stratification for the V-flame causes the suppression of the PVC instability. This explains the occasionally observed absence of the PVC under reacting conditions [8-10]. With the attempt to gain further insights into the interplay between the PVC, the density field and the flame, the authors investigated a bi-stable operating condition where the flame intermittently transitioned between the Vand M-shape. They tracked the flow field and flame shape during a V-M flame transition using high-speed PIV and OH-PLIF. They conjectured that the PVC plays a crucial role in the flame detachment process as it generates an unsteady stagnation point that destabilizes the flame root. Moreover it was found that the PVC induces additional mixing near the jet core that modifies the local density stratification in favor for the PVC, which potentially leads to a self-amplification process.

As an extension of the work of Oberleithner et al. [6], the present work now applies two novel methods of data analysis that are well adapted to the study of transient flame shape transitions: Firstly, the steady LSA is extended to the LSA of a time-varying mean flow. Secondly, the recently proposed spectral proper orthogonal decomposition (POD) is applied, which enables a better separation of different periodic instabilities than the conventional POD. Furthermore the present work now addresses the full bistable dynamics including both the V-M and M-V transitions.

\section{EXPERIMENTAL SETUP AND OPERATING CONDITION Gas turbine model combustor}

Measurements were performed at atmospheric pressure with the GT-typical PRECCINSTA swirl combustor based on a design by Turbomeca, which is shown schematically in Fig. 1. Perfectly premixed methane and air enter the cylindrical plenum $(d=78 \mathrm{~mm})$ and then pass through a swirl generator with 12 radial vanes. The swirling flow then reaches the combustion chamber through a burner nozzle $(d=27.85 \mathrm{~mm})$ with a conical bluff body. The chamber has a square cross-section of $85 \times 85 \mathrm{~mm}^{2}$ and a height of $114 \mathrm{~mm}$. Optical access to the chamber is provided by side walls made of quartz glass held by metal posts in the corners. The exit is composed of a conical part followed by

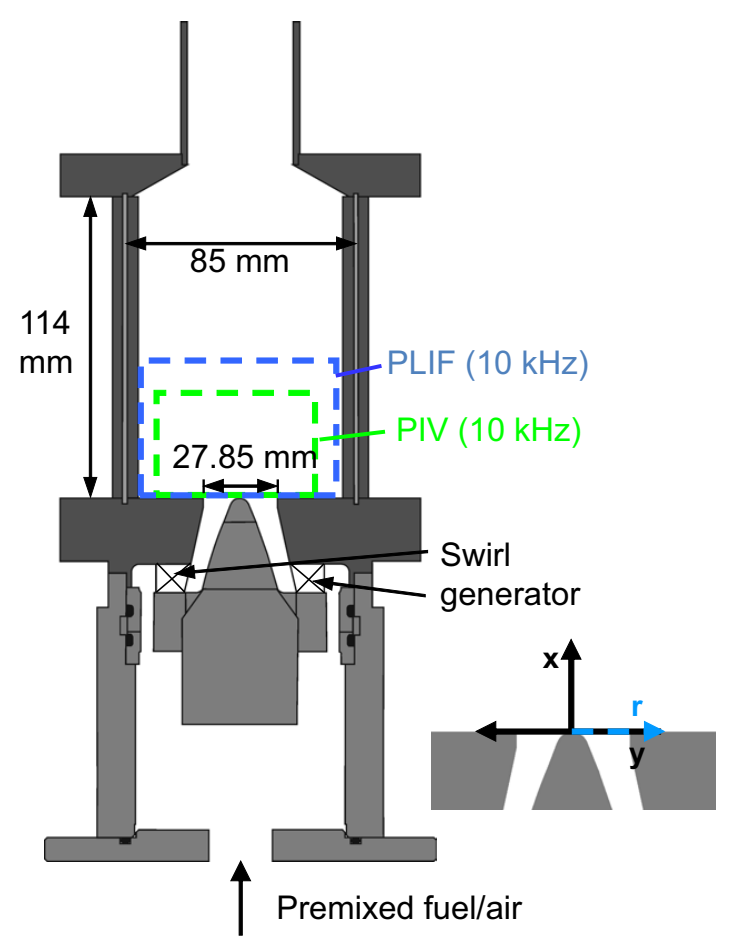

FIGURE 1. Schematics of gas turbine model combustor.

an exhaust duct $(d=40 \mathrm{~mm})$.

\section{Operating condition}

The previous work by Oberleithner et al. [6] showed that depending on the thermal power $P_{\text {th }}$ and equivalence ratio $\phi$, the flame can either assume an attached V-shape, a detached Mshape, or a bi-stable behavior alternating intermittently between $\mathrm{V}$ - and M-shape. For a fixed value of $P_{\mathrm{th}}, \mathrm{V}$-flames appear for larger, M-flames for smaller and bi-stable flames for intermediate values of $\phi$. The work further showed that M-flames exhibit a PVC whereas V-flames do not. The present study was performed for a bi-stable flame at $P_{\mathrm{th}}=20 \mathrm{~kW}$ and $\phi=0.7$. At this condition, the flame was alternating randomly about once per second between a V-shape without PVC and an M-shape with PVC.

\section{Measurement Techniques}

Time-resolved simultaneous stereo-PIV and OH-PLIF measurements with a repetition rate of $10 \mathrm{kHz}$ were performed using the experimental setup described in a recent publication [11]. The most important specifications are provided in the following. The stereo-PIV system employed a dual-cavity Nd:YAG laser (Edgewave IS-6IIDE, $2.6 \mathrm{~mJ} /$ pulse at $532 \mathrm{~nm}$ ) and $2 \mathrm{CMOS}$ cameras (LaVision HSS 8) equipped with Tokina lenses $(f=100 \mathrm{~mm}$, $f / 2.8)$ and bandpass filters $(532 \pm 1 \mathrm{~nm})$. OH-PLIF was excited at 
$283.2 \mathrm{~nm}$ by a dye laser (Sirah Credo, $80 \mu \mathrm{J} /$ pulse at $283.2 \mathrm{~nm}$ ) pumped with a Nd:YAG laser (Edgewave IS-8IIE, $4 \mathrm{~mJ} /$ pulse at $523 \mathrm{~nm}$ ). The OH-PLIF emission was recorded using an intensified CMOS camera (LaVision HSS 5 with HS-IRO) equipped with a Cerco UV lens $(f=45 \mathrm{~mm}, f / 1.8)$ and a bandpass filter (300-325 nm). The beams of the two laser systems were expanded into two coplanar vertical light sheets across the central section of the combustion chamber. About $4 \%$ of the UV light sheet was deflected into a cuvette filled with a fluorescent liquid for sheet profile imaging using an intensified CMOS camera (LaVision HSS 5 with HS-IRO) equipped with a Nikon lens ( $f=$ $50 \mathrm{~mm}$, set to $f / 4$ ). The measurement domains of PIV and OHPLIF are displayed in Fig. 1. For PIV the air flow was seeded with $\mathrm{TiO}_{2}$ particles with a diameter of about $1 \mu \mathrm{m}$. The sustained recording time was $0.8 \mathrm{~s}$ corresponding to 8000 single measurements. The PIV images were processed using a cross-correlation algorithm (LaVision DaVis 8.2) with a final interrogation window size of $16 \times 16$ pixel corresponding to $1.3 \times 1.3 \mathrm{~mm}^{2}$. The single-shot inhomogeneities and energy fluctuations of the PLIF laser sheet were corrected using the simultaneously recorded dye cuvette sheet profiles. The PIV particle images are further used to estimate the local gas density, which is required as an input of the LSA, according to the so-called quantitative light sheet (QLS) technique that derives the density from the Mie scattering signal of the PIV particles [6].

\section{DATA ANALYSIS METHODS}

\section{Spectral Proper Orthogonal Decomposition}

In order to survey the chronology of events during the measured transients, we employ a modal decomposition of the data reading

$$
\mathbf{u}(\mathbf{x}, t)=\overline{\mathbf{u}}(\mathbf{x})+\mathbf{u}^{\prime}(\mathbf{x}, t)=\overline{\mathbf{u}}(\mathbf{x})+\sum_{i=1}^{N} a_{i}(t) \Psi_{i}(x),
$$

which separates the fluctuating part of the velocity $\mathbf{u}^{\prime}$ into temporal coefficients $a_{i}$ and spatial modes $\Psi_{i}$. The modal basis is constructed using the recently introduced Spectral Proper Orthogonal Decomposition (SPOD) [12], which is an extension of the classical Proper Orthogonal Decomposition (POD) [13]. Both methods where very successfully used to extract the dominant coherent structures from PIV measurements of swirling jets. Usually, the POD identifies the dominant coherent structures [14], but in case of multiple interacting and intermittent modes the single flow structures are not properly assigned to single modes. The SPOD includes an additional spectral constraint that provides much better separation in such cases $[12,15]$. However, it should be noted that its application is limited to timeresolved measurements.
Here, a short overview of the SPOD approach is given. More detailed derivations can be found in [12]. To obtain the SPOD, the correlation matrix of the data set needs to be calculated between individual snapshots. The correlation matrix is computed as

$$
R_{i, j}=\frac{1}{N} \int_{V} \mathbf{u}^{\prime}\left(\mathbf{x}, t_{i}\right) \mathbf{u}^{\prime T}\left(\mathbf{x}, t_{j}\right) \mathrm{d} V
$$

where $N$ is the number of snapshots, $V$ specifies the measured domain over which the correlation is integrated and ()$^{T}$ indicates the transpose. In addition to the classical POD algorithm, the correlation matrix is filtered along all diagonals with a low-pass filter, resulting in a filtered correlation matrix $\mathbf{S}$, with the elements given as

$$
S_{i, j}=\sum_{k=-N_{f}}^{N_{f}} g_{k} R_{i+k, j+k} .
$$

The low-pass filter is implemented as a symmetric finite impulse response filter with a filter coefficients vector $\mathbf{g}$ of length $2 N_{f}+1$. A Gaussian filter is used to obtain a smooth response in time and frequency domain. The filter size $N_{f}$ is the central adjusting parameter of the SPOD that controls the spectral constraint of the modes. In the current investigation this is set to two periods of the PVC oscillation $\left(N_{f}=50 \approx 2 f_{P I V} / f_{P V C}\right)$, which is in line with the previous applications. Generally, there are little changes of the results for small variations of the filter size. As a rule of thump, the filter size should be at least as long as a single period of the lowest frequency mode but not to large to avoid splitting of flow structures into several modes. The latter is easily visible when the size is increased in moderate steps.

The temporal coefficients $\mathbf{a}_{i}=\left[a_{i}\left(t_{1}\right), \ldots, a_{i}\left(t_{N}\right)\right]^{T}$ and mode energies $\lambda_{i}$ are obtained from the eigenvectors and eigenvalues of the filtered correlation matrix.

$$
\mathbf{S a}_{i}=\lambda_{i} \mathbf{a}_{i} ; \quad \lambda_{1} \geq \lambda_{2} \geq \cdots \geq \lambda_{N} \geq 0
$$

The subscript $i$ refers to single eigenvalues, which are sorted in descending order. Naturally, the SPOD modes are sorted according to their kinetic energy content $\lambda_{i}$. This may sometimes hide less energetic modes among strong stochastic fluctuations. In the work of Sieber et al. [12] a ranking of the modes according to the spectral coherence of mode pairs is introduced. This is used to select relevant modes and graphically highlight periodic modes in the SPOD spectrum that will be shown later.

\section{Linear hydrodynamic stability analysis}

Within the framework of linear stability theory, the PVC is interpreted as a global hydrodynamic instability [6, 14, 16-18]. 
The term global implies that the entire flow dynamics are dominated by a single oscillatory mode. The most prominent example of a global instability is the von Kármán vortex street. Global modes are self-excited and are driven by an internal feed-back mechanism. The place where this feed-back occurs is called the wavemaker region. The determination of the wavemaker is particularly important as it determines the growth rate (amplitude) and frequency of the global mode.

In this study we employ a local linear stability analysis (LSA) to find the wavemaker and to determine the growth rate and frequency of the PVC. The current approach is well in line with the previous investigations of the stable $\mathrm{M}$ - and V-flame configuration and the reader is referred to this article for further details [6]. However, in contrast to the previous study, the analysis is now applied to a mean flow that evolves in time, which requires some additional considerations regarding the involved time scales. The reader is referred to a recent analysis of a transient isothermal swirling jet for detailed discussion of this concept [19].

For the LSA, the transient mean flow data is decomposed into four parts: the mean, the shift, the periodic (coherent) and the turbulent part, reading

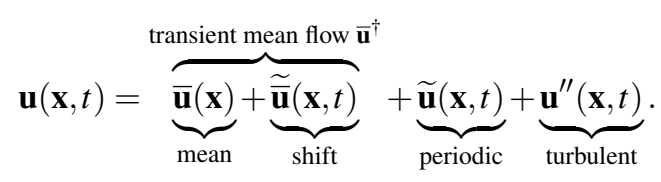

The governing equations are derived by substituting the decomposition $\mathbf{u}=\overline{\mathbf{u}}^{\dagger}+\widetilde{\mathbf{u}}$ into the Navier-Stokes and continuity equations. Linearization around the transient mean state results in the stability equations

$$
\begin{aligned}
\frac{\partial \widetilde{\mathbf{u}}}{\partial t}+(\widetilde{\mathbf{u}} \cdot \nabla) \overline{\mathbf{u}}^{\dagger}+\left(\overline{\mathbf{u}}^{\dagger} \cdot \nabla\right) \widetilde{\mathbf{u}} & =-\frac{1}{\bar{\rho}^{\dagger}} \nabla \widetilde{p}+\frac{1}{\operatorname{Re}} \Delta \widetilde{\mathbf{u}} \\
\nabla \cdot \widetilde{\mathbf{u}} & =0 .
\end{aligned}
$$

We assume that the transient mean state $\overline{\mathbf{u}}^{\dagger}$ is a quasi steady solution of the forced Navier-Stokes equations, as pursued by Mantič-Lugo et al. [20]. Therefore, the temporal changes of the mean flow are contributed to changes of the forcing by the Reynolds stresses. Hence, the timescale at which the mean flow changes is directly coupled to the timescale at which the coherent structure $\widetilde{\mathbf{u}}$ is amplified [19].

Assuming a quasi-parallel flow, the perturbation is decomposed into normal modes $\widetilde{\mathbf{u}}=\hat{\mathbf{u}} \mathrm{e}^{i(\alpha x+m \theta-\omega t)}$ and $\widetilde{p}=$ $\hat{p} \mathrm{e}^{i(\alpha x+m \theta-\omega t)}$, where $\alpha$ denotes the axial wave number, $m$ the azimuthal wave number and $\omega$ the frequency. Eqs. (6) and (7) together with the disturbance ansatz and appropriate boundary conditions yield an eigenvalue problem that is discretized with the Chebyshev pseudospectral collocation technique [21]. The resulting dispersion relation $\mathscr{D}(\omega, \alpha, m)=0$ is solved for complex $\alpha$ and complex $\omega$ and $m=1$. We then search for a saddlepoint in the mapping from $\alpha$ to $\omega$, which corresponds to the wave with zero group velocity. The growth rate of this wave is called absolute growth rate $\omega_{0}$ and it determines whether a flow profile is convectively or absolutely unstable [22]. The distinction between convective and absolute instability at every flow slice is an important information, because a finite region of absolute instability is necessary for the weakly non-parallel flow to sustain a global mode [23].

Once the absolute instability is determined, the wavemaker location is given by a frequency selection criterion [24]. For the M-flame configuration, the wavemaker is located at the combustor inlet [6] and the global mode growth rate $\sigma$ is given by the imaginary part of the absolute frequency at the this point, reading

$$
\sigma=\mathfrak{I}\left\{\omega_{0}(x=0)\right\}
$$

For $\sigma>0$ the flow is globally unstable and for $\sigma<0$ the flow is globally stable. The frequency of the PVC is given by the real part of the absolute frequency and has been shown to compare extremely well with the measurements [6].

Throughout this study, we assume that the wavemaker location does not change during the transient. This substantially simplifies the problem and we can track the PVC growth rate from a LSA at $x=0$. Moreover, we use a constant eddy viscosity to model the turbulent-coherent interactions in contrast to previous studies $[6,18,25]$. The calibration of a spatially and temporally varying eddy viscosity from the transient data introduces more uncertainty than the potential benefit. The current eddy viscosity is calibrated against the M-flame case to achieve zero growth of the global mode. This results in an effective Reynolds number in Eq. (6) of $\operatorname{Re}=\frac{U D}{v+v_{t}}=120$ with $v$ as the molecular viscosity and $v_{t}$ as the eddy viscosity.

As mentioned above, the LSA is based on a transient mean flow, which has to be properly estimated from the time-resolved fields of velocity and density. For this purpose the velocity and density fields are first smoothed temporally using a moving average filter with a span of $5 \mathrm{~ms}$. Then the data is symmetrized in $y$-direction, i.e., $\mathbf{u}_{\text {symm }}(x, y, t)=(\mathbf{u}(x, y, t)+\mathbf{u}(x,-y, t)) / 2$. The resulting fields of velocity and density that are used as input for the LSA are shown in Fig. 7.

\section{OVERALL CHARACTERISTICS OF THE FLOW AND FLAME TRANSIENT}

In this work the dynamics of the bi-stable flame is analyzed using one sustainted time-series of 8000 PIV and OH-PLIF measurements, corresponding to a duration of $800 \mathrm{~ms}$. The series includes one transition from $\mathrm{V}$ - to M-shape and one sub- 

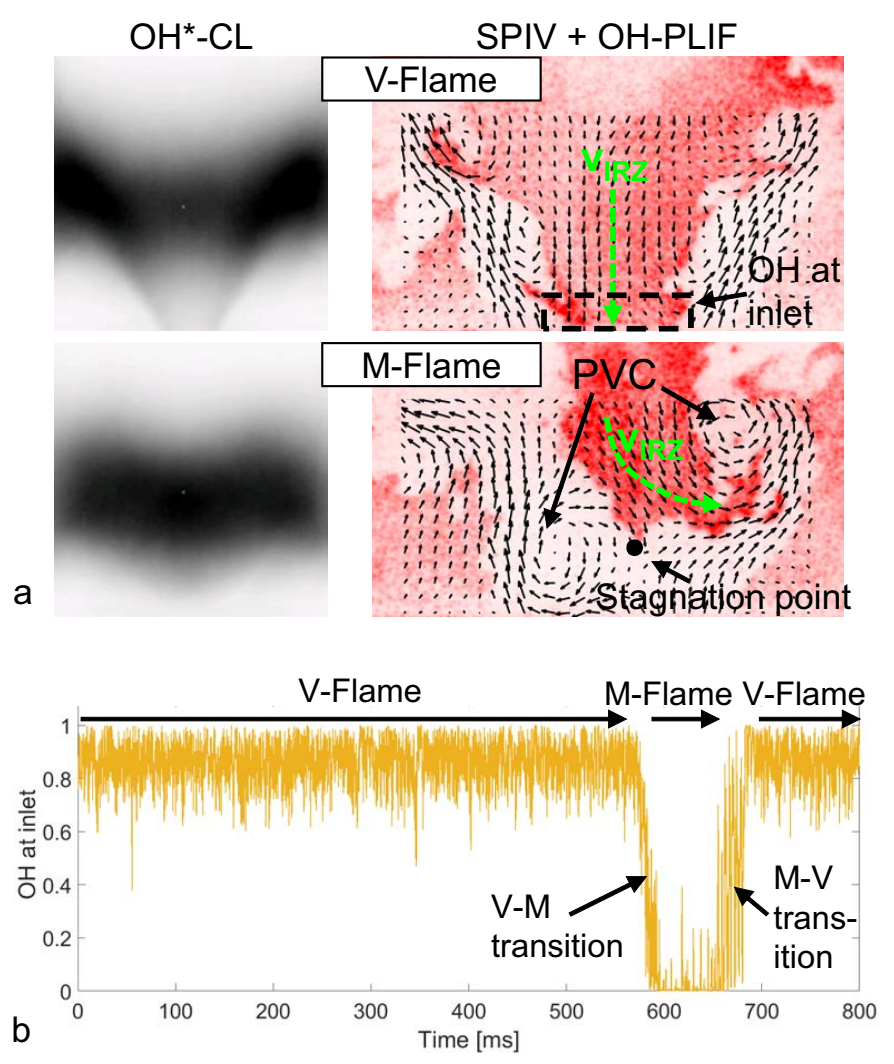

FIGURE 2. a) OH-chemiluminescence images and simultaneous PIV and OH-PLIF measurements during phases of V-and M-shape. b) Temporal dynamics of the $\mathrm{OH}$ signal at the inlet.

sequent transition back to $\mathrm{V}$-shape. Figure 2 a shows two $\mathrm{OH}-$ chemiluminescence images averaged during phases of $\mathrm{V}$ - and $\mathrm{M}$ shape, respectively. It is seen that the V-flame is attached at the burner nozzle at the bottom, whereas the M-flame is lifted by about $10 \mathrm{~mm}$. Further details of the V-and M-flame can be seen in the corresponding instantaneous PIV and OH-PLIF measurements on the right. While no major vortex structures appear for the V-flame, the flow field of the M-flame exhibits a distinct zigzag vortex pattern that represents the PVC [6]. This implies the presence of the stagnation point in the inner recirculation zone (IRZ), which stabilizes the M-flame at $x \approx 10 \mathrm{~mm}$. For the Vflame, by contrast, the flow in the IRZ goes straightly towards the nozzle at the bottom (green arrow).

The dynamics of the flame transitions is tracked using the OH-PLIF signal integrated over the zone near the chamber inlet (marked with the dashed rectangle). The resulting time-series is shown in Fig. $2 \mathrm{~b}$. The $\mathrm{OH}$-signal is high until $t \approx 570 \mathrm{~ms}$, which indicates an attached V-flame. The subsequent decrease of the $\mathrm{OH}$-signal indicates a lift-off of the flame, and the formation of a detached M-flame at $t \approx 600 \mathrm{~ms}$. Starting at $t \approx 650 \mathrm{~ms}$, the $\mathrm{OH}$ -
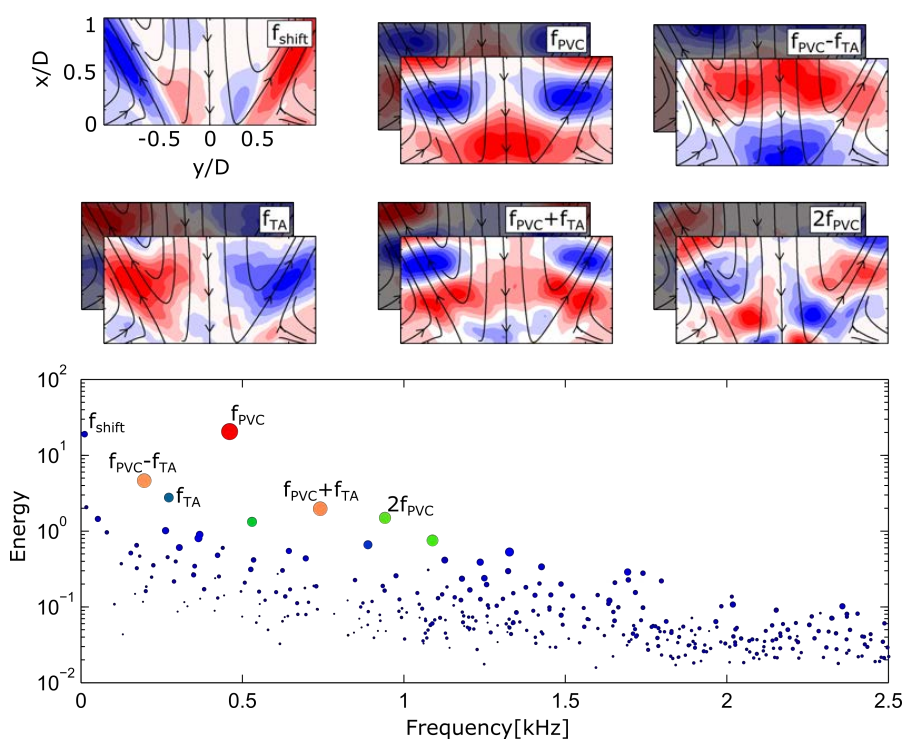

FIGURE 3. SPOD spectrum of the recorded transient. The dots represent the average frequency and energy of coupled SPOD modes and the size (and color) of the dots represents the spectral coherence. The most prominent modes are labeled and the corresponding mode shapes (transversal component) are shown in the images above. The mean flow direction is also indicated by streamlines.

signal increases again, indicating the transition back to a V-flame that remains until the end of the series.

The SPOD was applied to the snapshots recorded between $t=560$ and $700 \mathrm{~ms}$, which covers the entire detachment and reattachment process of the flame. Figure 3 shows the corresponding SPOD spectrum, where the modes are plotted according to their average frequency and relative energy content. The size (or color) of the dots represent the spectral coherence of the modes. From the spectrum we identify a few discrete modes that clearly stick out due to their energy content or due to their spectral coherence. The shapes of these modes are displayed in the frames above the SPOD spectrum showing the fluctuating transversal velocity component with superimposed streamlines of the mean flow field.

The mode with the strongest temporal coherence and the highest energy with $f_{\mathrm{PVC}}=460 \mathrm{~Hz}$ can be identified as the PVC. The mode shape shows a clear axisymmetry of the transversal component which corresponds to a helical instability with azimuthal wavenumber $m=1$. The SPOD also picks up the first higher harmonic of the PVC indicated by $2 f_{\mathrm{PVC}}$. The highly energetic mode at zero frequency is a shift mode. It represents the change of the mean flow field during the recorded sequence. At $f_{\mathrm{TA}}=275 \mathrm{~Hz}$ we find a relatively weak mode with poor temporal coherence. From the mode shape we can identify this mode as an axisymmetric flow oscillations. As the axisymmetric mode is not known to become self-excited in swirling jets, we conjecture that 


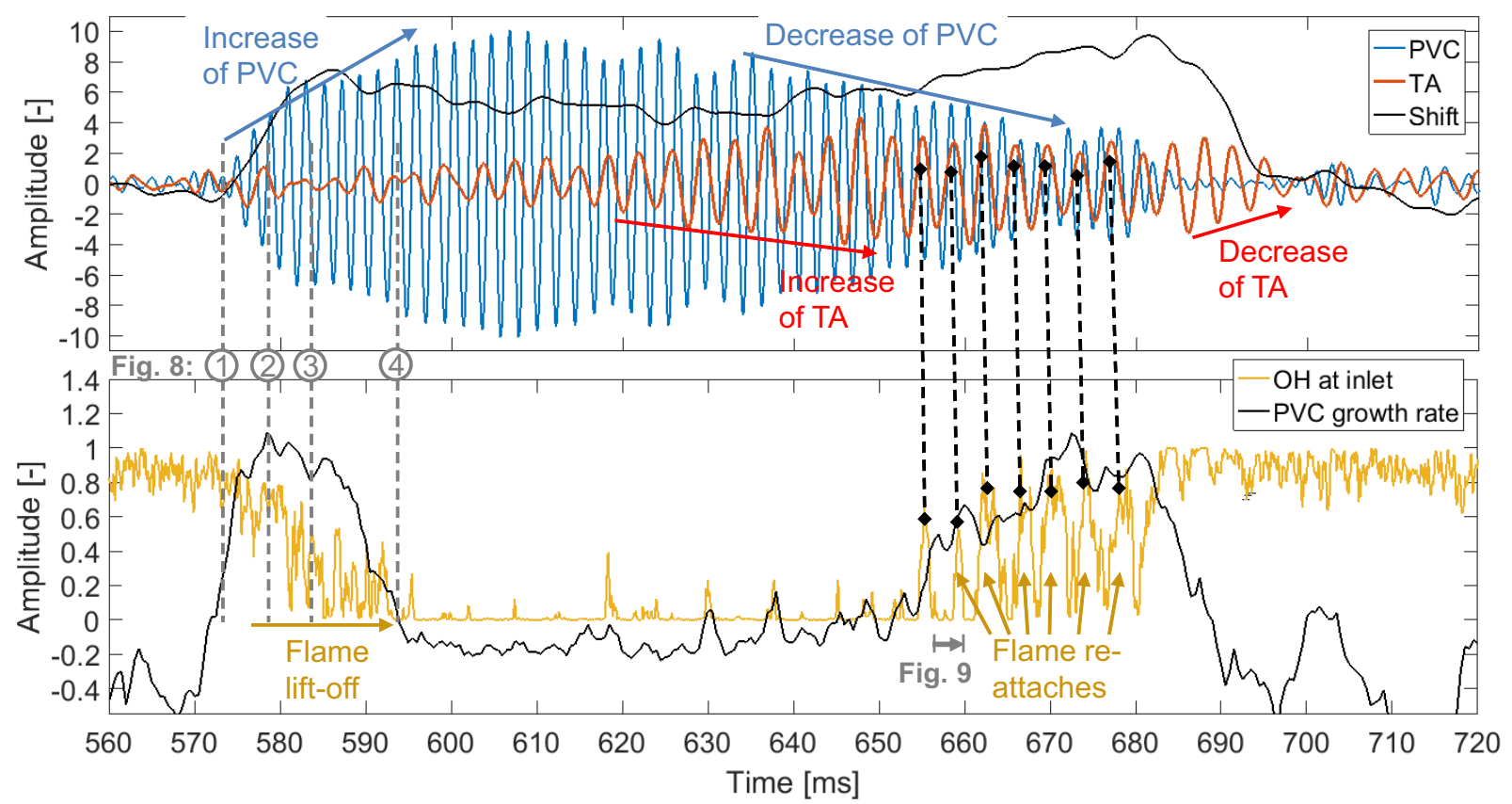

FIGURE 4. Dynamics of the V-M and M-V flame shape transition: The upper plot shows the SPOD coefficients of the PVC mode, the TA mode and the shift mode. The lower plot shows the $\mathrm{OH}$ signal at the inlet and the PVC growth rate $\sigma$ obtained from the LSA.

this mode is forced by a thermoacoustic (TA) instability. There are two strong additional modes at the sum and difference of the PVC frequency $f_{\mathrm{PVC}}$ and the frequency $f_{\mathrm{TA}}$. These modes show the same symmetry as the PVC and represent the interactions between the PVC and the mode at $f_{\mathrm{TA}}$.

The main dynamical features identified from the SPOD spectrum are the shift mode, the PVC, an axisymmetric mode (TA instability) and two modes that represent the interaction between the PVC and the TA instability. As a next step we consider the temporal evolution of the modes and relate it to the flame shape transition process. In Fig. 4 the SPOD mode coefficients are shown together with the integrated $\mathrm{OH}$ at the inlet indicating the flame at-/detachment as described above. The PVC oscillation sets in at about $570 \mathrm{~ms}$ and saturates at $600 \mathrm{~ms}$. Thereafter it continuously decays until it completely vanishes at about $680 \mathrm{~ms}$. The flame detachment appears to occur somewhat after the onset of the PVC. The flame remains detached until $650 \mathrm{~ms}$ and then behaves intermittently for another $30 \mathrm{~ms}$ before it ultimately reattaches at about $680 \mathrm{~ms}$, which coincides with the total suppression of the PVC. The shift mode changes simultaneously with the PVC amplitude but it levels off much earlier and remains approximately constant until $690 \mathrm{~ms}$. The TA mode starts to grow once the mean flow shift has terminated at about $590 \mathrm{~ms}$ and it continues growing until the mean flow shifts back to its initial shape at about $695 \mathrm{~ms}$. As already seen from the SPOD spectrum, the TA mode oscillates at a lower frequency than the PVC.

\section{STEPS OF V-M and M-V FLAME TRANSITIONS}

Based on the results from the SPOD, we postulate a sequence of events that describes the flame detachment and reattachment process. It will serve as a working hypothesis for the subsequent sections, where we investigate the mechanisms in more detail.

1. Slow drifts in the inflow conditions destabilize the PVC.

2. At the border of instability, a turbulent perturbation initiates the PVC.

3. Self-amplification sets in and the PVC evolves.

4. The PVC causes the flame to detach.

5. The PVC saturates at the limit-cycle and the flame stabilizes as an M-flame

6. The M-flame becomes TA unstable.

7. The TA oscillations suppresses the PVC.

8. The flame oscillates and reattaches to the inlet.

9. The attached V-flame is TA-stable and the oscillations die out. 


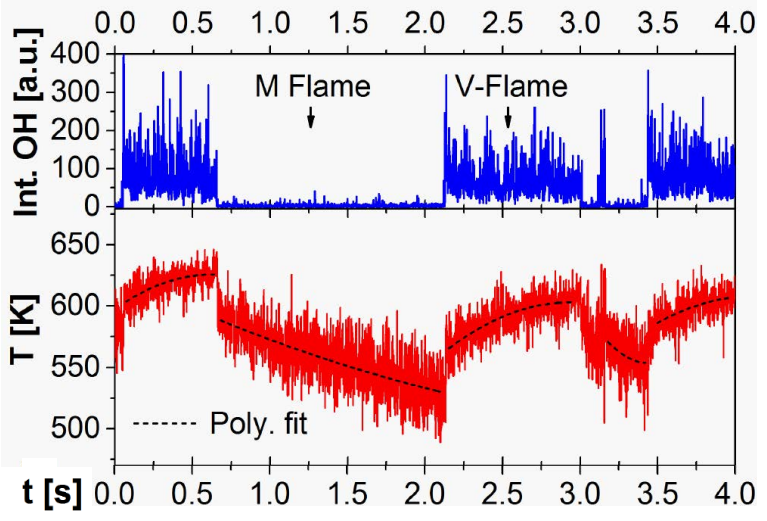

FIGURE 5. Temporal variation of bluff body temperature during bistable flame shape transitions, reprinted from Ref. [26]

\section{Step 1: Slow temperature and flow field changes}

When the flame changes its shape from $\mathrm{V}$ to $\mathrm{M}$ or vice versa, the heat load at different parts of the combustion chamber also changes. A recent experimental study for the present bi-stable flame using thermographic phosphors showed that this in turn leads to local changes of the temperature at the bluff body and base plate of the chamber [26]. An exemplary time-series from this study is plotted in Fig. 5. It is seen that the bluff body heats up by more than $50 \mathrm{~K}$ when the flame is attached, and cools down when the flame is detached.

The observed drifts of temperature may in turn induce slow drifts of the flow and density fields in the chamber. These drifts were quantified using linear fits of the local time-series of velocities $v_{x}$ and $v_{z}$ and density $\rho$ during the long time interval from $t=0 \mathrm{~ms}$ until the onset of the flame detachment at $t=568 \mathrm{~ms}$. The resulting drift of the axial velocity field is shown in Fig. 6 for $t=0,300$ and $568 \mathrm{~ms}$. It can be seen that the axial velocity field changes indeed, with the main difference being the increase of backflow in the IRZ (marked red). The corresponding drifts of $v_{z}$ and $\rho$ (not shown), on the other hand, were found to be much smaller.

To infer the possible effects on the stability of the PVC, we conduct a LSA of the drifting velocity and density fields at $t=0$, $100, \ldots, 500$ and $568 \mathrm{~ms}$. The resulting PVC growth rate at these times is plotted in Fig. 6. It is negative throughout indicating the global stability of the flow field prior to the V-M-transition, but it increases continuously in time and approaches the border of instability $(\sigma \approx 0)$ as we get closer to the start of the transition at $t=570 \mathrm{~ms}$. Since the main temporal changes were observed for the backflow velocity in the IRZ, this increase of growth rate is largely attributed to the drift of the backflow velocity. This agrees well with the study of Terhaar et al., who found that the PVC growth rate is largely determined by the backflow velocity [27].

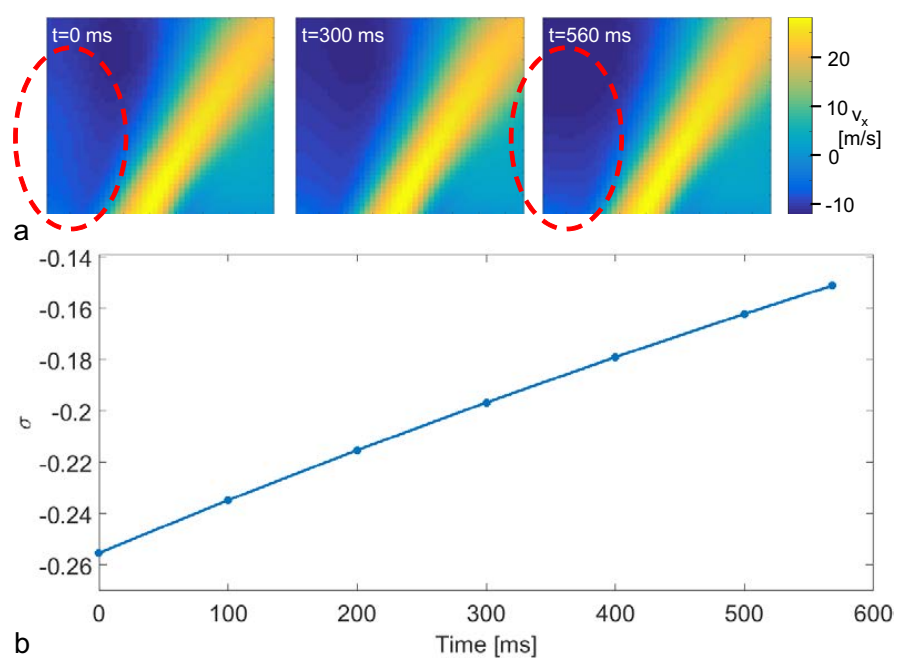

FIGURE 6. a) Variation of axial velocity field during the V-flame period $t=0-568 \mathrm{~ms}$ before the onset of transition. b) Corresponding variation of the PVC growth rate $\sigma$.

\section{Steps 2-3: Onset of the PVC}

Once the flow is at the border of instability (PVC growth rate $\sigma \approx 0$ ), a small variation of the inflow velocities may lead to a short-time destabilization of the PVC. In the previous investigation, a short-time appearance of the PVC was observed that was found to be correlated with a turbulent short-time increase of the inflow velocity and a lift-off of the flame [6]. It was conjectured in that study that the PVC is only sustained once it is strong enough to activate a self-amplifying process. In order to validate this hypothesis, we apply the LSA to the transient mean flow and track the PVC growth rate throughout the flame detachment process.

The role of the PVC in the V-M flame transition starting at $t \approx 570 \mathrm{~ms}$ is studied in the following using the time series plotted in Fig. 4 and the sequence of PIV and OH-PLIF images shown in Fig. 8. The times of the images in Fig. 8 are marked with dashed lines in Fig. 4. At $t=572.8 \mathrm{~ms}$, the V-shaped flame is well attached and no PVC is present as inferred from the low value of the SPOD PVC mode coefficient in Fig. 4. At $t=578.7$ $\mathrm{ms}$, the flame is still attached, but now a distinct PVC-like vortex pattern appears in the flow field, and the PVC mode coefficient has increased significantly. It is difficult to identify the specific event that caused the formation of the PVC since the flow is at the border of instability and thus even minor turbulent fluctuations of velocity or density, possibly out of the plane of measurement, may initiate the formation.

Comparing the PVC amplitude with the PVC growth rate shown in Fig. 4 within the time interval $t=570-580 \mathrm{~ms}$, we note that the amplitude of the PVC increases synchronously with the predicted growth rate. This is striking, as one would expect from 

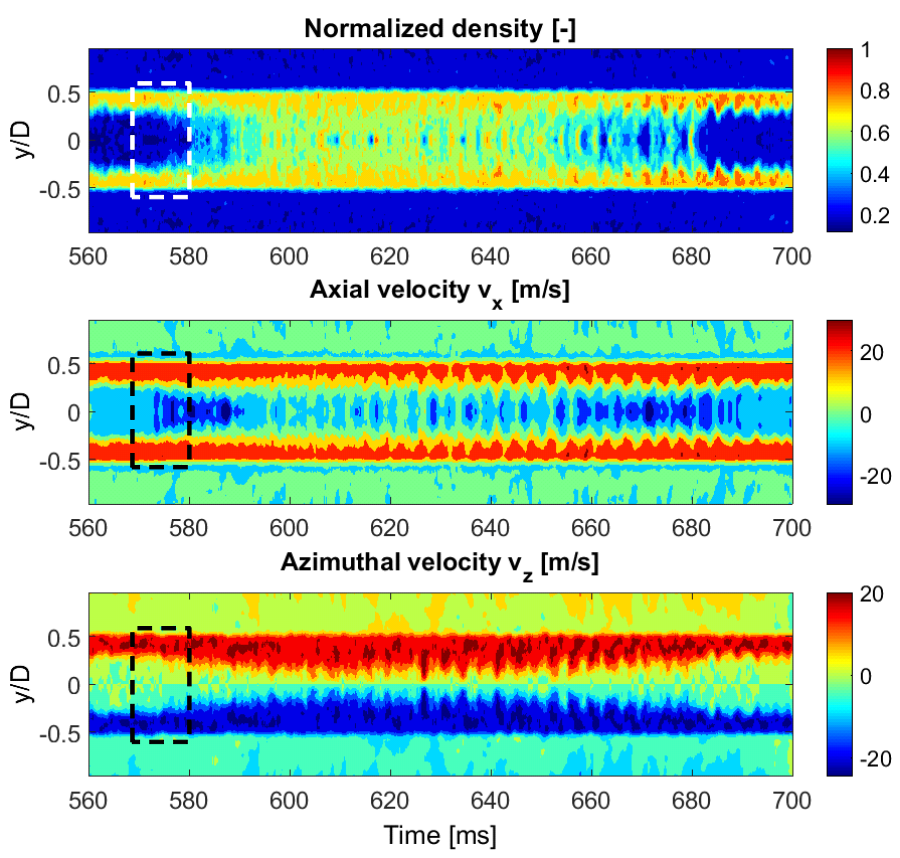

FIGURE 7. Temporally smoothed and radially symmetrized flow and density field at the inlet versus time.

mean field theory $[28,29]$ that the growth rate is positive and maximum prior to the onset of the PVC and it should decrease with increasing PVC amplitude. The increase of the growth rate with an increase of the PVC amplitude is strongly indicative for a self-amplification mechanism. To further study this mechanism, the temporal dynamics of profiles of density and velocity at the inlet $(x=0 \mathrm{~mm})$ that were used for the LSA is shown in Fig. 7. It is seen that during the phase $t=570-580 \mathrm{~ms}$, the axial velocity decreases and the stratification of density increases in the region around the centerline $(y=0 \mathrm{~mm})$. Computations based on a constant density field (not shown) indicate that both the modification of the density field and the velocity field contribute to the increase of the growth rate. The results from the transient LSA give credibility to the idea that the interaction between the PVC and the flow/density field induces a self-amplification process that helps to sustain the growth of the PVC. This allows the PVC to arise at subcritical or near critical conditions. The question whether additional mixing or flame lift-off is causing this favorable change of the mean flow and density field cannot be answered at this point.

\section{Steps 4-5: Flame detachment and saturation of the PVC limit-cycle}

After the PVC has reached a high amplitude at $t \approx 580 \mathrm{~ms}$, the attached V-flame starts to detach from the nozzle, as indicated by the decrease of $\mathrm{OH}$ signal plotted in Fig. 4. The image for
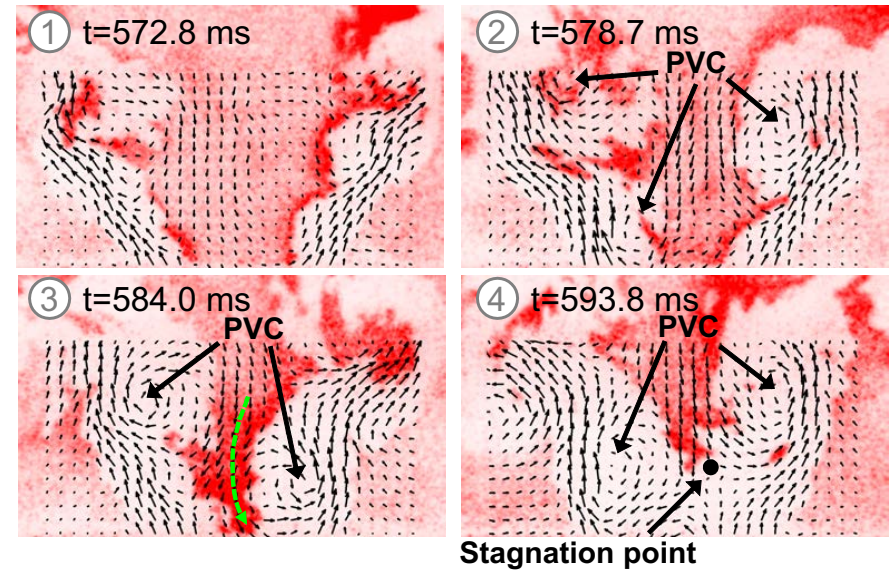

FIGURE 8. Sequence of PIV and OH-PLIF measurements showing the formation of the PVC and the subsequent detachment of the flame.

$t=584.0 \mathrm{~ms}$ in Fig. 8 shows that a strong PVC is present and the size of the IRZ has decreased significantly compared to $t=572.8$ ms. There is, however, still a continous backflow in the IRZ (green arrow) and therefore the flame is still attached. At $t=$ $593.8 \mathrm{~ms}$, the strength of the PVC has further increased and now a stagnation point appears in the IRZ. This blocks the backflow of hot burned gas towards the nozzle and thus stabilizes the flame base near this point.

The flame detachment process is completed at approximately $t=600 \mathrm{~ms}$ and the PVC reaches its maximum amplitude. The flow and flame dynamics have converged to a new stable condition, which is characterized by an M-shaped flame with a PVC. The saturation of the PVC occurs through a mean flow modification induced by coherent Reynolds stresses, which is reflected by the decrease of the global mode growth rate $\sigma$ to zero. The results from the LSA at the limit-cycle $(t=600-640$ $\mathrm{ms})$ are perfectly in line with our study of the stable M-flame [6] and confirm the saturation mechanism of this type of instability according to mean field theory $[28,29]$.

\section{Steps 6-7: Onset of TA instability and suppression of the PVC}

The plots of the SPOD coefficients in Fig. 4 reveal that once the flame has transitioned to a stable M-flame, a TA instability arises after $t=620 \mathrm{~ms}$ that goes along with the decay of the PVC oscillations. The dynamics of flow field and flame during one cycle of the TA instability is shown in Fig. 9. At $t=657.1$ $\mathrm{ms}$ the typical configuration of the M-flame with PVC and lifted flame base is present. At $t=658.2 \mathrm{~ms}$ the formation of a symmetric vortex pair above the inlet is observed that is likely caused by the pumping motion of the TA instability. The formation of the symmetric vortices goes along with the formation of a contin- 
uous backflow in the IRZ (green arrow). This enables the flame to propagate upstream as seen in the images at $t=658.6,659.1$ and $659.6 \mathrm{~ms}$. At $t=660.1 \mathrm{~ms}$ the symmetric vortex pair has decayed and the PVC vortex pattern is re-established, and the flame is lifted again. The comparison of TA mode coeffificients and $\mathrm{OH}$ signal in Fig. 4 during the phase $t=655-675 \mathrm{~ms}$ (marked with dashed lines) shows that a similar dynamics of transient flame re-attachement and detachement takes place repeatedly, and that this dynamics is directly coupled to the oscillation of the TA instability.

From the viewpoint of stability theory, the TA instability and the PVC may interact in two different ways according to Terhaar et al. [30]: They may either interact through a mean flow modification or through a mean flow modulation. The first mechanism implies that the perturbations induced by the TA instability are amplified in the shear layers to large-scale coherent structures that modify the mean flow as such that the PVC becomes linearly stable. This requires a strong convective shear layer instability (Kelvin-Helmholtz instability) at the frequency of the TA instability. The LSA can model this type of interaction, as it is based on the modified mean flow itself. Hence, the suppression of the PVC via this mechanism would be indicated by a reduction of the growth rate $\sigma$. This is not observed in the present case (see Fig. 4).

The mean flow modulation represents a direct nonlinear interaction between the PVC and the TA instability. It can bee understood as a modulation of the PVC growth rate and frequency at the TA instability frequency. In other words, the pressure fluctuations induced by the TA instability effectively modulate the massflow into the combustor and thereby the PVC dynamics. Peaks in the frequency spectrum with the sum and difference of the frequencies of the PVC and the TA instability as seen in the SPOD spectrum (Fig. 3) is a clear indicator of this mechanism [30,31].

Due to the negligence of the nonlinear terms, the LSA cannot account for the nonlinear interactions between the PVC and the TA instability. However, the PVC growth rates derived from the LSA give some indication that the suppression mechanism does indeed not occur via a mean flow modification. As seen in Fig. 4, the global mode growth rate $\sigma$ gets larger than zero at $t=650 \mathrm{~ms}$ and increases as the PVC amplitude decreases. This appears counter-intuitive at the first glance, but it can be explained by mean field theory. When the PVC grows to a finite amplitude (see Fig. 4; $t=570-600 \mathrm{~ms}$ ), it induces Reynolds stresses that act as a forcing on the mean flow. This forcing causes the saturation of the PVC as it modifies the mean flow as such that the PVC growth rate reduces to zero $(t=600-650$ $\mathrm{ms}$ ). Once the PVC gets suppressed by the TA instability, the Reynolds stress forcing and corresponding mean flow correction are suppressed as well. This is reflected by a positive PVC growth rate $(t=670 \mathrm{~ms})$. If one would, theoretically, switch off the TA instability at this point, the PVC would immediately grow

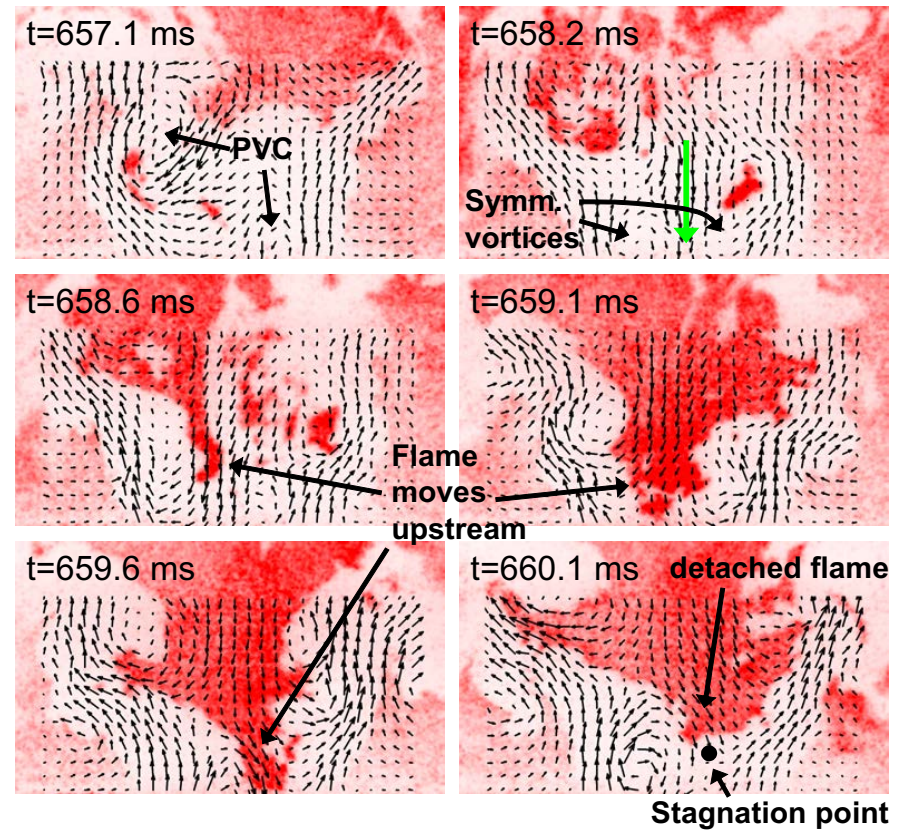

FIGURE 9. Sequence of PIV and OH-PLIF measurements showing the transient attachment and detachment of the flame during one cycle of the TA instability.

again at the growth rate that is given by the LSA.

\section{Steps 8-9: The reattachment process}

As described above, the increased amplitude of the TA instability after $t=655 \mathrm{~ms}$ leads to periodic flame re-attachement and detachement. At the same time, the PVC amplitude further decreases and reaches a near zero value at $t=685 \mathrm{~ms}$. Therefore, the process of detachement, which is induced by the PVC, is no longer sustained and the flame remains attached after $t=685$ ms. It is further seen that after the attached V-flame has stabilized, the TA instability dies out after $t=700 \mathrm{~ms}$, which is most likely attributed to the change of the convective time-delays.

\section{SUMMARY AND CONCLUSIONS}

The flame shape transition mechanisms of a turbulent swirling bi-stable flame have been studied using time-resolved simultaneous stereo-PIV and OH-PLIF measurements, spectral proper orthogonal decompostion (SPOD) and linear stability analysis (LSA). The flame alternates intermittently between an $\mathrm{M}$-shape featuring a precessing vortex core (PVC) and a V-shape without PVC, which implies that the PVC is repeatedly formed and suppressed. It was further observed that a thermoacoustic (TA) instability arises and decays temporarily.

The starting point of the V-M-transition is a slow drift of ve- 
locity and density fields during the phase with V-flame that are likely caused by slow variations of temperature within the combustor. The LSA showed that these drifts lead to an increase of the (negative) growth rate of the PVC. When the growth rate is close to zero, a random turbulent event can trigger the formation of the PVC, which then undergoes a self-amplification and reaches a limit-cycle. The PVC in turn leads to the formation of an unsteady stagnation point in the IRZ that causes the lift-off of the flame, which then assumes an M-shape.

The M-V-transition is governed by completely different mechanisms: After the flame has assumed the M-shape, a TA instability develops. The pumping motion of the TA instability leads to a periodic formation of symmetric ring vortices at the nozzle exit, and to a decay of the PVC. The symmetric vortices temporarily suppress the stagnation point in the IRZ and thus lead to periodic short-time re-attachment and subsequent lift-off of the flame. When the PVC has decreased sufficiently, the flame remains attached and assumes a $\mathrm{V}$-shape. When the formation of the V-flame is completed the TA instability decays again.

The results show that the dynamics of turbulent bi-stable swirl flames is governed by complex interactions of TA and hydrodynamic instabilities, and slow temperature-induced drifts of velocity and density. The better understanding of these interactions obtained in this work may support the design of improved swirl combustors with enlarged ranges of stable operation.

\section{ACKNOWLEDGMENT}

The authors from DLR gratefully acknowledge funding from the DLR project IVTAS. Authors from TU Berlin would like to thank for financial support from the Research Association for Combustion Engines (FVV) and funding from the German Research Foundation (DFG) under project grants PA 920/30-1.

\section{REFERENCES}

[1] Fritsche, D., Füri, M., and Boulouchos, K., 2007. “An experimental investigation of thermoacoustic instabilities in a premixed swirl-stabilized flame". Combustion and Flame, 151, pp. 29-36.

[2] Biagioli, F., Güthe, F., and Schuermans, B., 2008. "Combustion dynamics linked to flame behaviour in a partially premixed swirled industrial burner". Experimental Thermal and Fluid Science, 32, pp. 1344-1353.

[3] Tummers, M.J., Hbner, A., van Veen, E.H., H. K., and van der Meer, T., 2009. "Hysteresis and transition in swirling nonpremixed flames". Combustion and Flame, 156, pp. 447-459.

[4] Renaud, A., Ducruix, S., Scouflaire, P., and Zimmer, L., 2015. "Flame shape transition in a swirl stabilised liquid fueled burner". Proceedings of the Combustion Institute, 35, p. 33653372.
[5] Guiberti, T., Durox, D., Scouflaire, P., and Schuller, T., 2015. "Impact of heat loss and hydrogen enrichment on the shape of confined swirling flames". Proceedings of the Combustion Institute, 35, pp. 1385-1392.

[6] Oberleithner, K., Stöhr, M., Seong, H. I., and Arndt, Christoph M.and Steinberg, A. M., 2015. "Formation and flame-induced suppression of the precessing vortex core in a swirl combustor: Experiments and linear stability analysis". Combustion and Flame, 162(8), pp. 3100-3114.

[7] Hermeth, S., Staffelbach, G., Gicquel, L. Y., Anisimov, V., Cirigliano, C., and Poinsot, T., 2014. "Bistable swirled flames and influence on flame transfer functions". Combustion and Flame, 161(1), pp. $184-196$.

[8] Selle, L., Lartigue, G., Poinsot, T., Koch, R., Schildmacher, K.-U., Krebs, W., Prade, B., Kaufmann, P., and Veynante, D., 2004. "Compressible large eddy simulation of turbulent combustion in complex geometry on unstructured meshes". Combustion and Flame, 137, pp. 489-505.

[9] Freitag, M., and Janicka, J., 2007. "Investigation of a strongly swirled unconfined premixed flame using les". Proceedings of the Combustion Institute, 31, pp. 14771485.

[10] De, A., Zhu, S., and Acharya, S., 2010. "An experimental and computational study of a swirl-stabilized premixed flame". Journal of Engineering for Gas Turbines and Power, 132, p. 071503.

[11] Stöhr, M., Yin, Z., and Meier, W., 2016. "Interaction between velocity fluctuations and equivalence ratio fluctuations during thermoacoustic oscillations in a partially premixed swirl combustor". Proceedings of the Combustion Institute, 36, in press, doi: 10.1016/j.proci.2016.06.084.

[12] Sieber, M., Paschereit, C. O., and Oberleithner, K., 2016. "Spectral proper orthogonal decomposition". Journal of Fluid Mechanics, 792, 4, pp. 798-828.

[13] Holmes, P., Lumley, J., and Berkooz, G., 1998. Turbulence, Coherent Structures, Dynamical Systems and Symmetry. Cambridge Monographs on Mechanics. Cambridge University Press, Cambridge [England].

[14] Oberleithner, K., Sieber, M., Nayeri, C. N., Paschereit, C. O., Petz, C., Hege, H.-C., Noack, B. R., and Wygnanski, I., 2011. "Three-dimensional coherent structures in a swirling jet undergoing vortex breakdown: stability analysis and empirical mode construction". Journal of Fluid Mechanics, 679, pp. 383-414.

[15] Sieber, M., Paschereit, C. O., and Oberleithner, K., 2016. "Advanced Identification of Coherent Structures in SwirlStabilized Combustors". Journal of Engineering for Gas Turbines and Power, 139(2), p. 021503.

[16] Gallaire, F., Ruith, M., Meiburg, E., Chomaz, J.-M., and Huerre, P., 2006. "Spiral vortex breakdown as a global mode". Journal of Fluid Mechanics, 549, pp. 71-80.

[17] Juniper, M. P., 2012. "Absolute and convective instability in 
gas turbine fuel injectors". In Proceedings of ASME Turbo Expo, GT2012-68253, pp. 189-198.

[18] Tammisola, O., and Juniper, M. P., 2016. "Coherent structures in a swirl injector at re $=4800$ by nonlinear simulations and linear global modes". Journal of Fluid Mechanics, 792, 003, pp. 620-657.

[19] Rukes, L., Sieber, M., Paschereit Oliver, C., and Oberleithner, K., 2015. "Transient evolution of the global mode in turbulent swirling jets: experiments and modal stability analysis". European Journal of Mechanics - B-Fluids. (under review).

[20] Mantič-Lugo, V., Arratia, C., and Gallaire, F., 2015. "A self-consistent model for the saturation dynamics of the vortex shedding around the mean flow in the unstable cylinder wake". Physics of Fluids, 27(7), p. 074103.

[21] Khorrami, M. R., Malik, M. R., and Ash, R. L., 1989. “Application of spectral collocation techniques to the stability of swirling flows". J. Comput. Phys., 81(1), pp. 206-229.

[22] Huerre, P., and Monkewitz, P. A., 1990. "Local and global instabilities in spatially developing flows". Annual Review of Fluid Mechanics, 22, pp. 473-537.

[23] Chomaz, J. M., Huerre, P., and Redekopp, L. G., 1988. "Bifurcations to local and global modes in spatially developing flows". Physical Review Letters, 60, pp. 25-28.

[24] Chomaz, J.-M., Huerre, P., and Redekopp, L. G., 1991. "A frequency selection criterion in spatially developing flows". Stud. Appl. Math., 84, pp. 119-144.

[25] Rukes, L., Paschereit Oliver, C., and Oberleithner, K., 2016. "An assessment of turbulence models for linear hydrodynamic stability analysis of strongly swirling jets". $E u$ ropean Journal of Mechanics - B/Fluids, 59, pp. 205-218.

[26] Yin, Z., Nau, P., and Meier, W., 2016. "Responses of combustor surface temperature to flame shape transitions in a turbulent bi-stable swirl flame". Experimental Thermal and Fluid Science, in press, doi: 10.1016/j.expthermflusci.2016.11.004.

[27] Terhaar, S., Oberleithner, K., and Paschereit, C., 2015. "Key parameters governing the precessing vortex core in reacting flows: An experimental and analytical study". Proceedings of the Combustion Institute, 35(3), pp. 3347 3354.

[28] Noack, B. R., Afanasiev, K., Morzyński, M., Tadmor, G., and Thiele, F., 2003. "A hierarchy of low-dimensional models for the transient and post-transient cylinder wake". Journal of Fluid Mechanics, 497, pp. 335-363.

[29] Barkley, D., 2006. "Linear analysis of the cylinder wake mean flow". Europhysics Letters, 75, pp. 750-756.

[30] Terhaar, S., Ćosić, B., Paschereit, C., and Oberleithner, K., 2016. "Suppression and excitation of the precessing vortex core by acoustic velocity fluctuations: An experimental and analytical study". Combustion and Flame, 172, pp. 234251.
[31] Moeck, J. P., Bourgouin, J.-F., Durox, D., Schuller, T., and Candel, S., 2012. "Nonlinear interaction between a precessing vortex core and acoustic oscillations in a turbulent swirling flame". Combustion and Flame, 159(8), pp. 26502668. 